\title{
Prognostic implications of immunosuppressive protein expression in tumors as well as immune cell infiltration within the tumor microenvironment in gastric cancer
}

\author{
Jin Won Kim $\cdot$ Kyung Han Nam $\cdot$ Sang-Hoon Ahn $\cdot$ Do Joong Park $\cdot$ Hyung-Ho Kim \\ Se Hyun Kim · Hyun Chang · Jeong-Ok Lee • Yu Jung Kim • Hye Seung Lee • \\ Jee Hyun Kim • Soo-Mee Bang · Jong Seok Lee $\cdot$ Keun-Wook Lee
}

Received: 17 June 2014/ Accepted: 28 October 2014/Published online: 26 November 2014

(c) The International Gastric Cancer Association and The Japanese Gastric Cancer Association 2014

\begin{abstract}
Background There are few data on the clinical implications of immunosuppressive protein expression in tumors and immune cell infiltration within the tumor microenvironment in patients with gastric cancer (GC).

Methods In this study, 243 patients with curatively resected GC were included. The levels of immunosuppressive protein expression [programmed cell death 1 ligand 1 (PDL1), cytotoxic T-lymphocyte antigen 4 (CTLA-4), and indoleamine 2,3-dioxygenase (IDO)] in tumors and the densities of immune cells [CD3(+), CD4(+), CD8(+), or PD$1(+)$ cells] within the tumor microenvironment were measured using immunohistochemical analysis.

Results Positive PD-L1, CTLA-4, and IDO expression was observed in 43.6, 65.8, and $47.7 \%$ of the patients, respectively. Expression of PD-L1, CTLA-4, and IDO was related to less advanced stage, intestinal type, and well/ moderately differentiated adenocarcinoma $(P<0.05)$. PDL1 expression was related to better disease-free survival
\end{abstract}

J. W. Kim and K. H. Nam contributed equally to this work.

Electronic supplementary material The online version of this article (doi:10.1007/s10120-014-0440-5) contains supplementary material, which is available to authorized users.

J. W. Kim $\cdot$ S. H. Kim $\cdot$ J.-O. Lee $\cdot$ Y. J. Kim

J. H. Kim · S.-M. Bang · J. S. Lee · K.-W. Lee $(\bowtie)$

Division of Hematology and Medical Oncology, Department of Internal Medicine, Seoul National University Bundang Hospital,

Seoul National University College of Medicine, Seongnam,

Republic of Korea

e-mail: hmodoctor@snubh.org

K. H. Nam · H. S. Lee

Department of Pathology, Seoul National University Bundang

Hospital, Seoul National University College of Medicine,

Seongnam, Republic of Korea
(DFS) and overall survival (OS) in GC [PD-L1(+) vs. PDL1(-) tumors: 5-year DFS rate, 82.6 vs. $66.9 \%$; 5-year OS rate, 83.0 vs. $69.1 \%(P$ values $<0.05)$ ]. Survival outcomes were also better in patients with a higher density of $\mathrm{CD} 3(+)$ cells within the tumor microenvironment than in those with a lower density of CD3(+) cells [5-year DFS rate, 80.9 vs. $67.0 \%$; 5-year OS rate, 82.5 vs. $68.0 \%$ $(P$ values $<0.05)$ ]. In multivariate analysis, these two immune markers had a prognostic impact on survival, independent of other clinical variables.

Conclusions GC patients with immunosuppressive protein expression (PD-L1, CTLA-4, or IDO) had distinct clinicopathological characteristics. PD-L1(+) expression and a high-CD3 tumor microenvironment are favorable prognostic markers in GC.

Keywords PD-L1 - PD-1 - CD3 - Tumor microenvironment $\cdot$ Gastric cancer

\section{Introduction}

Evading immune surveillance is a hallmark of biological capabilities in cancer development [1]. This immune

K. H. Nam

Department of Pathology, Haeundae Paik Hospital, Inje

University College of Medicine, Busan, Republic of Korea

S.-H. Ahn · D. J. Park · H.-H. Kim

Department of Surgery, Seoul National University Bundang

Hospital, Seoul National University College of Medicine,

Seongnam, Republic of Korea

H. Chang

Department of Hematology and Medical Oncology, International

St. Mary's Hospital, Incheon, Republic of Korea 
surveillance, by which the host immune system can recognize and destroy cancer cells, has been reported in animals and in human cancer patients, supporting the hypothesis that immune evasion by cancer cells plays an important role in the development and progression of tumors [2]. An immunogenic interaction exists between the host and the tumor, and the ability of the tumor to evade immune recognition often determines the clinical course of the disease [3]. Recently, immunotherapeutic agents targeting immunosuppressive proteins such as cytotoxic $\mathrm{T}$ lymphocyte-associated antigen-4 (CTLA-4), programmed cell death 1 (PD-1), programmed cell death 1 ligand 1 (PD-L1), and killer cell Ig-like receptor have been investigated as potential treatments for cancer [4]. Among these immunotherapeutic agents, antiCTLA-4, anti-PD-1, and anti-PD-L1 antibodies have shown promising clinical efficacy towards melanoma as well as several other cancer types [5-9].

Tumor-infiltrating immune cells have been identified in various malignant tumors, suggesting that immunological parameters may be significant and useful for assessing the prognosis of patients with cancer [10-12]. It has been reported that immunosuppressive proteins such as PD-L1, CTLA-4, and indoleamine 2,3-dioxygenase (IDO) are also present in tumors. On the surface, the presence of immune cells around the tumor seems to be a host response to the tumor, and the expression of immunosuppressive proteins in cancer appears to help the tumor to evade host immune surveillance. However, the clinical implications of the existence of immunosuppressive proteins in tumors or immune cells in the tumor microenvironment are still controversial, and their potential as prognostic markers is unclear, as this potential appears to vary with the study population used and methods employed. In addition, the functional or causal relationship between immunosuppressive proteins in tumors and immune cells in the tumor microenvironment has not yet been clearly defined in gastric cancer (GC).

Therefore, we compared clinicopathological findings with the expression levels of immunosuppressive proteins in tumors and the presence of immune cells in the tumor microenvironment in patients with GC. We also analyzed the association between immunosuppressive proteins in tumors and immune cells in the tumor microenvironment and investigated their potential for use as prognostic markers.

\section{Materials and methods}

\section{Patients}

A total of 289 patients with GC underwent gastric resection between May 2003 and December 2004 at Seoul National
University Bundang Hospital (SNUBH). Of these 289 patients, patients with stage IV cancer $(N=14)$ and those with insufficient tissue for analysis $(N=32)$ were excluded; 243 patients were finally included in this study. All patients received curative resection with D2 lymph node dissection. Clinical information was retrieved from electronic medical records (EMR). The median follow-up duration was 74 months (range 0-123 months). When the patient's date of death could not be verified through the EMR, it was checked using legitimate services from the Ministry of Public Administration and Security in Korea. This study was approved by the institutional review board of SNUBH (B-1311/226-301).

\section{Tissue microarrays}

Surgical specimens were used. All tissue samples were fixed in $10 \%$ buffered formalin for $24-48 \mathrm{~h}$ and then embedded in paraffin. Representative cores $(2 \mathrm{~mm}$ in diameter) were taken at random from the tumor blocks and arranged in new tissue array blocks using a trephine apparatus (SuperBioChips Laboratories, Seoul, Korea). An adequate case was defined as a tumor occupying $>10 \%$ of the core area. For each case, the location of an individual core in relation to the center or the invasive front of the tumor was not recorded. Non-neoplastic gastric mucosa specimens were included in each of the array blocks, and the tissue array blocks contained up to 60 cores. The expression levels of PD-L1, CTLA-4, IDO, PD-1, and CD3 were measured in all 243 patients. The expression levels of CD4 and CD8 in immune cells were analyzed in only 220 of the patients due to a lack of remaining tumor tissues in the other patients.

Immunohistochemical analysis of immunosuppressive proteins in tumors

PD-L1, CTLA-4, and IDO were selected as immunosuppressive proteins in tumors. The expression of these immunosuppressive proteins in tumors was evaluated via immunohistochemical analysis. In brief, $4-\mu$ m-thick sections from array blocks were deparaffinized in xylene and rehydrated through graded concentrations of ethanol. Antigen retrieval was then performed by immersing the slides in citrate buffer ( $\mathrm{pH}$ 6.0) and microwaving them for $10 \mathrm{~min}$. Nonspecific staining was blocked using $1 \%$ horse serum in Tris-buffered saline ( $\mathrm{pH} \mathrm{6.0)}$ for $3 \mathrm{~min}$. Commercially available primary antibodies were used according to the manufacturer's instructions (anti-PD-L1, rabbit polyclonal, 1:1500 dilution, Abcam, Cambridge, MA, USA; anti-CTLA-4, rabbit polyclonal, 1:200 dilution, Abcam; anti-IDO, mouse monoclonal, 1:500 dilution, Abcam). Antibody binding was detected using an avidin- 
biotin-peroxidase complex (Universal Elite $\mathrm{ABC}$ kit PK6200; Vectastain, Burlingame, CA, USA) for $10 \mathrm{~min}$ and diaminobenzidine tetrahydrochloride solution (Kit HK1535 K; Biogenex, San Ramon, CA, USA).

The expression of PD-L1 was predominantly observed in the cytoplasm and plasma membrane of tumor cells. CTLA-4 and IDO were expressed in the cytoplasm of tumor cells. The immunoreactivity of PD-L1, CTLA-4, and IDO was evaluated semiquantitatively on the basis of the percentage and intensity of positive cells. The intensity of the staining was graded as negative, weak, moderate, or strong. When $>10 \%$ of tumor cells showed moderate to strong staining intensity for PD-L1, CTLA-4, or IDO, the case was recorded as positive for each protein (Fig. S1 in the Electronic supplementary material, ESM).

Density of immune cells in the tumor microenvironment

The densities of CD3(+), CD4(+), CD8(+), and PD-1(+) cells in the tumor microenvironment were assessed using a previously described image analysis system [13, 14]. The following primary antibodies were used: anti-CD3, rabbit polyclonal (1:100 dilution), Dako, Glostrup, Denmark; anti-CD4, rabbit monoclonoal (ready to use), clone SP35, Ventana Medical Systems, Tucson, AZ, USA; anti-CD8, mouse monoclonal (ready to use), clone C8/144B, Dako, Carpinteria, CA, USA; anti-PD-1, clone EPR4877(2), rabbit monoclonal (1:300 dilution), Epitomics, Burlingame, CA, USA. Briefly, stained slides were scanned at a magnification of $200 \times$ using the ScanScope CS2 (Aperio Technologies, Vista, CA, USA), and the scanned images were analyzed with the ImageScope software (version 12.0.0.5039, Aperio Technologies) to quantify the positive cells in each tumor region (both epithelial and stromal compartments), and then graded as negative, weak $(1+)$, moderate $(2+)$, or strong $(3+)$. Measurements were recorded as the number of positive cells $(2+$ or $3+)$ per unit of tissue surface area (in square millimeters). Cases were classified into two groups according to the median values of the measurements [median CD3(+) cell density, $662.86 / \mathrm{mm}^{2}$; median CD4(+) cell density, $408.84 / \mathrm{mm}^{2}$; median $\mathrm{CD} 8(+)$ cell density, $375.48 / \mathrm{mm}^{2}$; median PD$1(+)$ cell density, $42.88 / \mathrm{mm}^{2}$ ]. These median values were cut-off points for high-CD3 (vs. low-CD3), high-CD4 (vs. low-CD4), high-CD8 (vs. low-CD8), and high-PD-1 (vs. low-PD-1) cases (Fig. S1 of the ESM).

\section{Statistical analysis}

The association between clinicopathological findings and the expression of immunosuppressive proteins in tumors or the density of immune cells in the tumor microenvironment was evaluated using the $\chi^{2}$ test or a linear-by-linear association. Survival was analyzed using Kaplan-Meier plots and log-rank tests. Disease-free survival (DFS) was measured from the date of gastrectomy to tumor relapse, death from any cause (before tumor relapse), or the last followup. Overall survival (OS) was measured from the time of the operation to death from any cause or the last follow-up. A $P$ value of $<0.05$ was considered significant. The clinical variables with $P<0.05$ in univariate analysis were included in a multivariate analysis with the Cox regression model. Statistical analysis was performed with IBM SPSS Statistics 20 for Windows.

\section{Results}

Patient characteristics

The baseline patient characteristics are shown in Table 1. Of the 243 patients, $62.6 \%$ were men, and $21.8 \%$ were aged $>70$ years. Most patients underwent subtotal gastrectomy $(79.0 \%)$. Intestinal and diffuse types (according to the Lauren classification) comprised 46.1 and $46.5 \%$ of the cancers, respectively. Cancer stage included stage I (49.4\%), stage II (16.9\%), and stage III (33.8 \%). Adjuvant chemotherapy was administered in $36.6 \%$ of the patients.

Clinicopathological findings according

to the expression of immunosuppressive proteins in tumors and the density of immune cells in the tumor microenvironment

Positive expression of PD-L1, CTLA-4, and IDO was identified in $43.6,65.8$, and $47.7 \%$ of the cases, respectively (Table 2). The expression of these proteins did not differ according to sex, age, or tumor location. In terms of histology, tumors that were positive for PD-L1, CTLA-4, and IDO were frequently identified in well to moderately differentiated adenocarcinoma $(55.3,75.4$, and $62.3 \%$, respectively). According to the Lauren classification, the expression of PDL1, CTLA-4, and IDO was higher in the intestinal type than in the diffuse type $(P=0.003, P=0.003$, and $P<0.001$, respectively). Although lymphatic invasion did not show a correlation with expression of PD-L1, CTLA-4, or IDO ( $P=0.067, P=0.324, P=0.112$, respectively), tumors without neural invasion revealed more positivity for CTLA4 than tumors with neural invasion $(P=0.031)$. Absence of vascular invasion was associated with $\operatorname{IDO}(+)$ tumors $(P=0.012)$. Tumors in less advanced stages exhibited more positivity for PD-L1, CTLA-4, and IDO $(P=0.047$, $P=0.016$, and $P=0.007$, respectively).

In the tumor microenvironment, the densities of immune cells $[\mathrm{CD} 3(+)$ or PD-1(+) cells] did not differ 
according to sex, age, lymphatic invasion, vascular invasion, neural invasion, or tumor location. The diffuse type (based on the Lauren classification) showed a higher density of $\mathrm{CD} 3(+)$ immune cells than the intestinal type $(P=0.021)$. Tumors with signet ring cell carcinoma or poorly differentiated histology according to the WHO classification showed more PD-1(+) immune cells than other histological types $(P=0.019)$. The density of CD3(+) and PD-1(+) immune cells was not associated with tumor stage. Additionally, a higher density of CD4(+) or CD8(+) cells was observed in poorly differentiated or signet ring cell carcinoma than in other histological types ( $P=0.049$ and $P=0.016$, respectively). The diffuse type showed a higher density of $\mathrm{CD} 8(+)$ immune cells than the intestinal type $(P=0.002)$. The absence of lymphatic or neural invasion was associated with a high density of $\mathrm{CD} 4(+)$ immune cells $(P<0.001$ and $P=0.005$, respectively). Tumors in less advanced stages exhibited a higher density of CD4(+) immune cells but not CD8(+) cells (Table S1 in the ESM, $P<0.001$ and $P=0.428$, respectively).

Correlation between immunosuppressive proteins in tumors and density of immune cells in the tumor microenvironment

We found no correlation between the expression of immunosuppressive proteins in tumors and the density of immune cells in the tumor microenvironment. As shown in Table 3, PD-L1(+) tumors comprised $41.3 \%$ of the tumors in high-CD3 microenvironments and $45.9 \%$ of the tumors in low-CD3 microenvironments $(P=0.472)$; there was also no correlation between the expression of PD-L1 in tumors and the density of CD4(+) or CD8(+) immune cells in the tumor microenvironment. The rate of PD-L1(+) tumors also did not differ according to the density of PD-1 in the tumor microenvironment $(P=0.177)$. Similarly, the expression of CTLA-4 and IDO in tumors did not differ according to the density of immune cells in the tumor microenvironment. However, there was a strong correlation among the expression of PD-L1, CTLA-4, and IDO in tumors (Table S2 of the ESM, $P<0.001)$. A strong correlation was also found among the densities of CD3, CD4, CD8, and PD-1 (Table S3 of the ESM, $P<0.001$ ).

Prognosis according to the expression of immunosuppressive proteins in tumors and the density of immune cells in the microenvironment

The 5-year DFS and OS rates of patients with PD-L1(+) tumors were 82.6 and $83.0 \%$, respectively, which were
Table 1 Baseline characteristics $(N=243)$

\begin{tabular}{|c|c|c|}
\hline Variable & No. of patients & $\%$ \\
\hline \multicolumn{3}{|l|}{ Sex } \\
\hline Male & 152 & 62.6 \\
\hline Female & 91 & 37.4 \\
\hline \multicolumn{3}{|l|}{ Age (median 62 , range $28-87$ years) } \\
\hline$<70$ & 190 & 78.2 \\
\hline$\geq 70$ & 53 & 21.8 \\
\hline \multicolumn{3}{|l|}{ Operation } \\
\hline Subtotal gastrectomy & 192 & 79.0 \\
\hline Total gastrectomy & 51 & 21.0 \\
\hline \multicolumn{3}{|l|}{ WHO classification } \\
\hline Well to moderately differentiated & 114 & 46.9 \\
\hline Poorly differentiated & 86 & 35.4 \\
\hline Signet ring cell & 33 & 13.6 \\
\hline Mucinous & 8 & 3.3 \\
\hline Unclassified & 2 & 0.8 \\
\hline \multicolumn{3}{|l|}{ Lauren classification } \\
\hline Intestinal & 112 & 46.1 \\
\hline Diffuse & 113 & 46.5 \\
\hline Mixed & 18 & 7.4 \\
\hline \multicolumn{3}{|l|}{ Lymphatic invasion } \\
\hline Absent & 119 & 49.0 \\
\hline Present & 124 & 51.0 \\
\hline \multicolumn{3}{|l|}{ Vascular invasion } \\
\hline Absent & 218 & 89.7 \\
\hline Present & 25 & 10.3 \\
\hline \multicolumn{3}{|l|}{ Neural invasion } \\
\hline Absent & 157 & 64.6 \\
\hline Present & 86 & 35.4 \\
\hline \multicolumn{3}{|l|}{ Stage by AJCC7 } \\
\hline IA & 94 & 38.7 \\
\hline IB & 26 & 10.7 \\
\hline IIA & 22 & 9.1 \\
\hline IIB & 19 & 7.8 \\
\hline IIIA & 22 & 9.1 \\
\hline IIIB & 27 & 11.1 \\
\hline IIIC & 33 & 13.6 \\
\hline \multicolumn{3}{|l|}{ Adjuvant chemotherapy } \\
\hline Yes & 89 & 36.6 \\
\hline No & 154 & 63.4 \\
\hline
\end{tabular}

significantly higher than those of patients without PD-L1 expression [66.9 and $69.1 \%$, respectively $(P<0.05)$; Fig. 1a, b]. Similarly, patients with CTLA-4(+) or $\mathrm{IDO}(+)$ tumors demonstrated numerically better DFS $[P=0.380$ (Fig. 1c) and $P=0.624$ (Fig. 1e), respectively] and $\operatorname{OS}[P=0.448$ (Fig. 1d) and $P=0.618$ (Fig. 1f), respectively], although these findings were not statistically significant. Patients with tumors with high- 


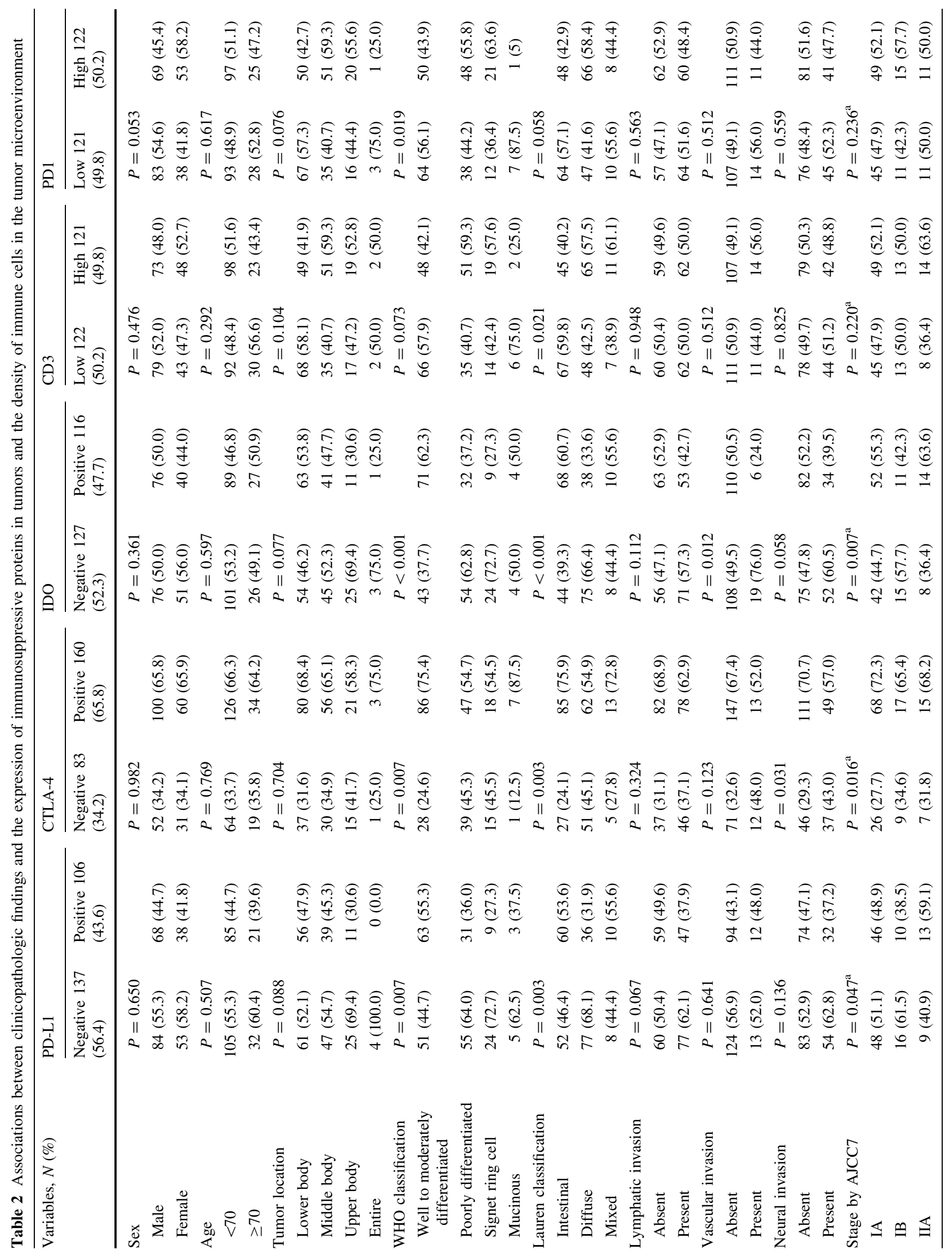




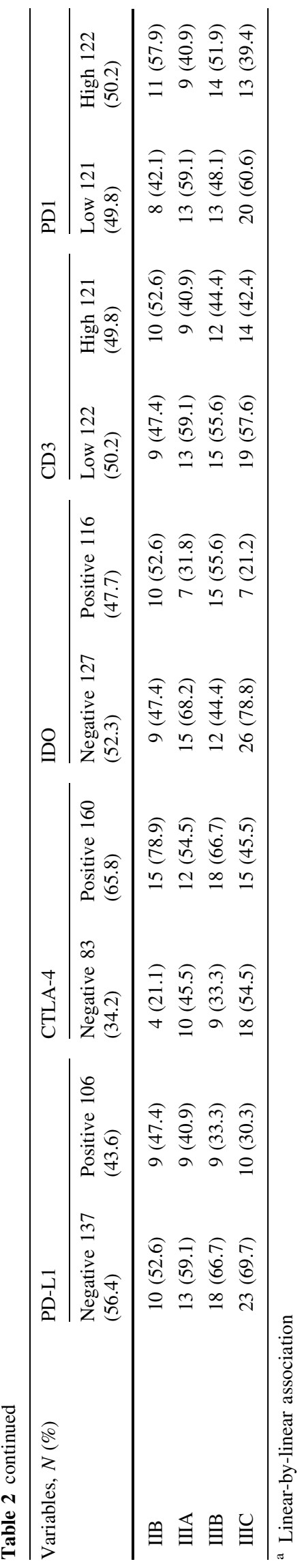

CD3 microenvironments had longer DFS and OS than those with tumors with low-CD3 microenvironments [5year DFS rate, 80.9 vs. $67.0 \%(P=0.006$, Fig. 2a); 5-year OS rate, 82.5 vs. $68.0 \%(P=0.002$, Fig. 2b)]. Regarding $\mathrm{CD} 4(+)$ and $\mathrm{CD} 8(+)$ immune cells, highCD4 or high-CD8 microenvironments showed better DFS $[P=0.004$ (Fig. 2c) and $P=0.111$ (Fig. 2e), respectively $]$ and $\mathrm{OS} \quad[P=0.003 \quad$ (Fig. 2d) and $P=0.043$ (Fig. 2f), respectively]. Tumors with highPD-1 microenvironments also demonstrated better prognoses, but this finding was not statistically significant (Figs. 2g, h).

A multivariate analysis including age, lymphatic invasion, vascular invasion, neural invasion, stage, PDL1 expression in tumors, and the density of CD3(+) cells in the tumor microenvironment-which were significant factors in univariate analyses-was performed. The positive expression of PD-L1 in tumors was an independently significant factor for improved DFS [hazard ratio $(\mathrm{HR})=0.582, P=0.018$ ], although this had only marginal significance in relation to $\mathrm{OS}$ (HR $=0.651, P=0.059)$. A high-CD3 tumor microenvironment was also independently related to better prognosis in both DFS and OS [HR $=0.614$ $(P=0.038)$ and $\mathrm{HR}=0.538(P=0.005)$, respectively] Among other factors, age $\geq 70$ years and advanced stage were significant independent risk factors for DFS; age $\geq 70$ years, vascular invasion, and advanced stage were identified as independent risk factors for OS in multivariate analysis (Table 4).

Separate multivariate analyses were also carried out in which the density of CD4(+) or CD8(+) immune cells was incorporated as an independent variable instead of the density of CD3(+) cells. However, in contrast to CD3(+) cells in the microenvironment, the densities of CD4 or CD8 immune cell subtypes did not have an independent prognostic value in these multivariate analyses (data not shown).

\section{Discussion}

This study was conducted to investigate the clinical implications of immunosuppressive protein expression in tumors and immune cell infiltration within the tumor microenvironment in GC patients. Our study included a larger number of patients $(N=243)$ than previous studies $[15,16]$. To increase the reliability of the method of detection, PD-L1 and other immunosuppressive proteins (CTLA-4 and IDO) in tumors were measured simultaneously. $\mathrm{CD} 3(+), \mathrm{CD} 4(+), \mathrm{CD} 8(+)$, and PD-1 (+) immune cells in the tumor microenvironment were also evaluated. 
Table 3 Association between the expression of immunosuppressive proteins in tumors and the density of immune cells in the tumor microenvironment

\begin{tabular}{|c|c|c|c|c|c|c|}
\hline \multirow[t]{2}{*}{ Variables } & \multicolumn{2}{|l|}{ PD-L1 } & \multicolumn{2}{|l|}{ CTLA-4 } & \multicolumn{2}{|l|}{ IDO } \\
\hline & Negative & Positive & Negative & Positive & Negative & Positive \\
\hline CD3 & $P=0.472$ & & $P=0.651$ & & $P=0.221$ & \\
\hline Low & $66(54.1 \%)$ & $56(45.9 \%)$ & $40(32.8 \%)$ & $82(67.2 \%)$ & $59(48.4 \%)$ & $63(51.6 \%)$ \\
\hline High & $71(58.7 \%)$ & $50(41.3 \%)$ & $43(35.5 \%)$ & $78(64.5 \%)$ & $68(56.2 \%)$ & $53(43.8 \%)$ \\
\hline CD4 & $P=0.171$ & & $P=0.091$ & & $P=0.686$ & \\
\hline Low & $65(59.6 \%)$ & $44(40.4 \%)$ & $40(36.7 \%)$ & $69(63.3 \%)$ & $56(51.4 \%)$ & $53(48.6 \%)$ \\
\hline High & $56(50.5 \%)$ & $55(49.5 \%)$ & $29(26.1 \%)$ & $82(73.9 \%)$ & $54(48.6 \%)$ & $57(51.4 \%)$ \\
\hline CD8 & $P=0.597$ & & $P=0.730$ & & $P=0.500$ & \\
\hline Low & $63(56.8 \%)$ & $48(43.2 \%)$ & $36(32.4 \%)$ & $75(67.6 \%)$ & $58(52.3 \%)$ & $53(47.7 \%)$ \\
\hline High & $58(53.2 \%)$ & $51(46.8 \%)$ & $33(30.3 \%)$ & $76(69.7 \%)$ & $52(47.7 \%)$ & $57(52.3 \%)$ \\
\hline PD-1 & $P=0.177$ & & $P=0.856$ & & $P=0.178$ & \\
\hline Low & $63(52.1 \%)$ & $58(47.9 \%)$ & $42(34.7 \%)$ & $79(65.3 \%)$ & $58(47.9 \%)$ & $63(52.1 \%)$ \\
\hline High & $74(60.7 \%)$ & $48(39.3 \%)$ & $41(33.6 \%)$ & $81(66.4 \%)$ & $69(56.6 \%)$ & $53(43.4 \%)$ \\
\hline
\end{tabular}

Detection of immunosuppressive markers including PDL1, CTLA-4, and IDO has been reported for various tumor types [4, 15-27]. In our study, the positive expression of PD-L1, CTLA-4, and IDO was noted in 43.6, 65.8, and $47.7 \%$ of the cases, respectively. This rate of positivity for PD-L1 in our study is similar to that seen in a previous study on GC, in which PD-L1 was detected in $42.2 \%$ of cases, although the expression was evaluated in a small number of patients with GC using different antibodies against PD-L1 $[15,16]$. In our study, high PD-L1 expression in GC was related to favorable clinicopathological features and prognosis (Fig. 1a, b; Table 4). In contrast to our observations, a previous study reported that high PDL1 expression in tumors was associated with poor prognosis and adverse clinicopathological findings in GC [15, 16]; various other tumors with PD-L1(+) expression have also been reported to have poor prognoses in some studies $[23,28-30]$. However, the prognostic impact of PD-L1 expression in patients with cancer remains controversial to date. Some studies have suggested that PD-L1 expression is not associated with a worse prognosis $[18,19,31]$. These different conclusions drawn by different studies may be explained by the small numbers of patients evaluated, the different antibodies used to detect PD-L1, and the different specimens used (frozen vs. paraffin-embedded tissues). To increase the reliability of the method of detection in our study, commercial antibodies were used, and other immunosuppressive proteins (CTLA-4 and IDO) were also evaluated simultaneously. Although there was no statistical significance, better prognoses were also noted for patients with CTLA-4 and IDO expression in tumors.

The overexpression of immunosuppressive proteins in a tumor can be attributed to either the response of the tumor to the endogenous host immunity or intrinsic pathways of the tumor itself. Immunosuppressive protein expression in tumors was shown to be correlated with tumor-infiltrating lymphocytes (TILs) in the case of ovarian cancer [23]. Interferon gamma was found to induce PD-L1 expression in breast cancer cells [17]. These reports support the idea that the expression of immunosuppressive proteins in tumors is induced as a response to endogenous host immunity. On the other hand, loss of phosphatase and tensin homolog and activation of the phosphatidylinositol3-OH kinase resulted in increased PD-L1 expression in human gliomas [32]. It was also reported that basal-type breast cancer with high PD-L1 had higher STAT1 levels [17], suggesting that overexpression of immunosuppressive proteins in tumors is associated with intrinsic pathways of the tumor itself. Our study showed that the protein expression levels of immunosuppressive proteins (PD-L1, CTLA-4, and IDO) in tumors were strongly correlated; however, the expression levels of immunosuppressive proteins in tumors were not related to the densities of immune cells $[\mathrm{CD} 3(+), \mathrm{CD} 4(+), \mathrm{CD} 8(+)$, and PD-1(+) cells] in the tumor microenvironment. These results support the idea that the expression of immunosuppressive proteins in GC is induced by intrinsic pathways of the tumor itself rather than as a response to endogenous host immunity. Additionally, in our study, tumors with positive expression of PD-L1, CTLA-4, and IDO proteins shared common clinical characteristics, such as less advanced stage, well/moderately differentiated histology, intestinal type, and lack of vascular invasion, which are known to be related to favorable prognosis. The better prognosis of GC patients who show the expression of immunosuppressive proteins in tumors may be explained by these observations from our study.

TILs have been reported to be associated with good prognosis in several tumor types [10, 12, 33, 34]. In our study, the density of CD3(+) TILs, including CD4(+) and 

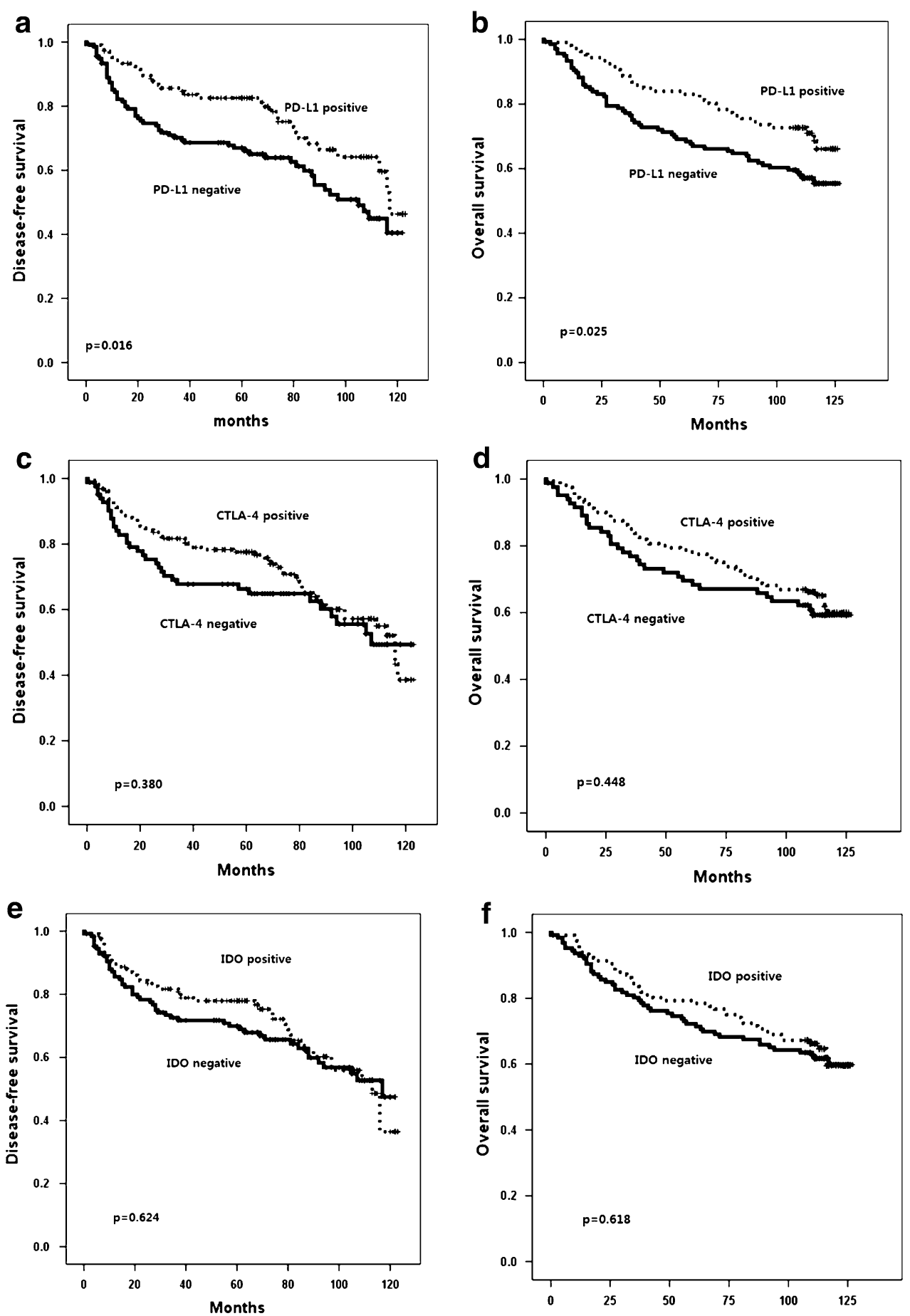

Fig. 1 Disease-free survival (DFS) and overall survival (OS) of patients with gastric cancer based on the expression of immunosuppressive proteins in tumors. a PD-L1(+/-), DFS. b PD-L1(+/-), OS. c CTLA-4(+/-), DFS. d CTLA-4(+/-), OS. e IDO(+/-), DFS. f IDO(+/-), OS

CD8(+) cells, was evaluated to account for immune cells in the tumor microenvironment. In concordance with previous reports [33, 34], tumors with increased levels of CD3 $(+)$ immune cells showed better DFS and OS $(P$ values $<0.05$; Fig. 2a, b); this prognostic impact of $\mathrm{CD} 3(+)$ cells was independently maintained in multivariate analysis (Table 4). Regarding CD4(+) or CD8(+) immune cells in the tumor microenvironment, univariate analyses showed that patients with a high density of $\mathrm{CD} 4(+)$ or $\mathrm{CD} 8(+)$ cells also had favorable survival outcomes compared with patients with a low density of CD4(+) or CD8(+) cells (Fig. 2c-f). However, the prognostic impact of CD4(+) or $\mathrm{CD} 8(+)$ cells in the microenvironment was not valid in multivariate analyses (data not shown). This finding 
Fig. 2 Disease-free survival (DFS) and overall survival (OS) of patients with gastric cancer according to the density of immune cells around the tumor. a High/low CD3(+), DFS. b High/low CD3(+), OS. c High/low CD4(+), DFS. d High/low CD4(+), OS. e High/low CD8 $(+)$, DFS. f High/low CD8(+), OS. g High/low PD-1(+), DFS. h High/low PD-1(+), OS
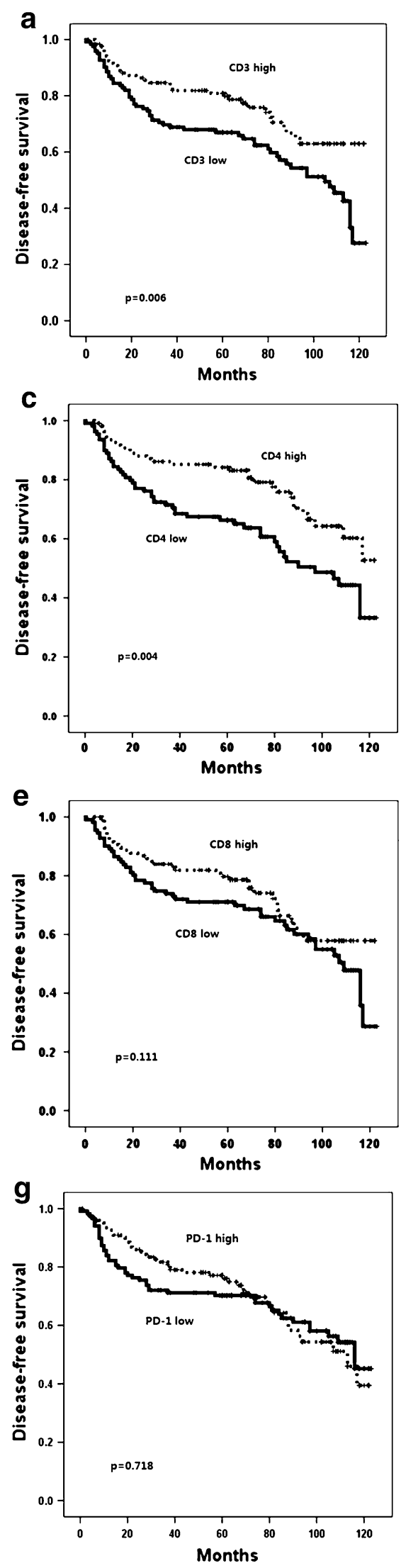
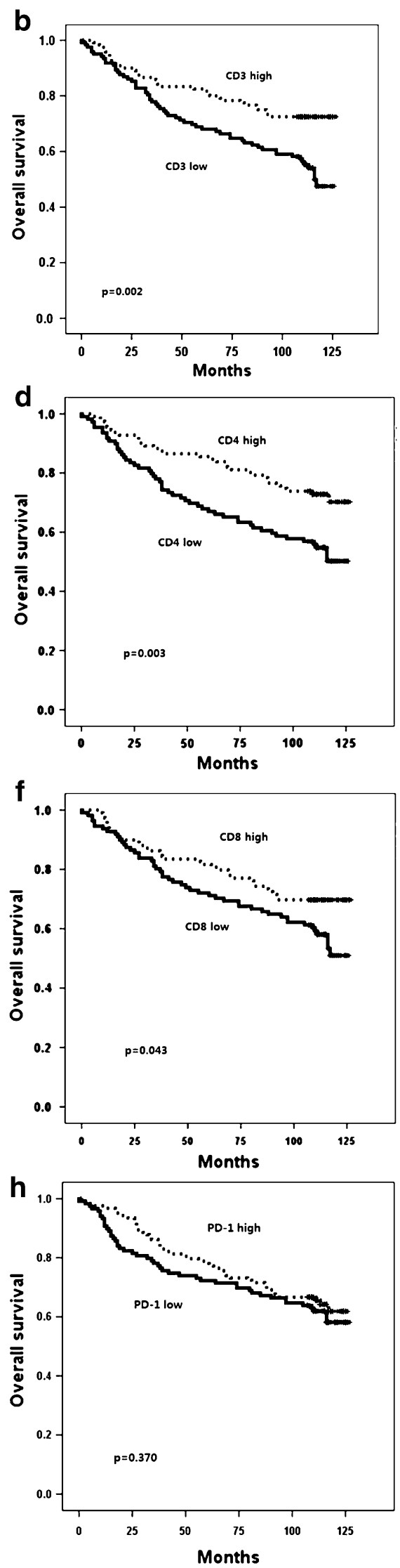
Table 4 Cox multivariable analysis of the independent risk factors for recurrence and death of patients with gastric cancer

\begin{tabular}{|c|c|c|c|c|c|c|}
\hline \multirow[t]{2}{*}{ Variables } & \multicolumn{3}{|c|}{ Disease-free survival } & \multicolumn{3}{|c|}{ Overall survival } \\
\hline & $\begin{array}{l}\text { Hazard } \\
\text { ratio }\end{array}$ & $\begin{array}{l}95 \% \text { confidence } \\
\text { interval }\end{array}$ & $P$ value & $\begin{array}{l}\text { Hazard } \\
\text { ratio }\end{array}$ & $\begin{array}{l}95 \% \text { confidence } \\
\text { interval }\end{array}$ & $P$ value \\
\hline \multicolumn{3}{|l|}{ Age } & $<0.001$ & & & $<0.001$ \\
\hline$<70$ & 1 & & & 1 & & \\
\hline$\geq 70$ & 2.459 & $(1.566-3.862)$ & & 2.727 & $(1.740-4.275)$ & \\
\hline \multicolumn{3}{|c|}{ Lymphatic invasion } & 0.396 & & & 0.993 \\
\hline Absent & 1 & & & 1 & & \\
\hline Present & 1.265 & $(0.735-2.178)$ & & 1.002 & $(0.589-1.707)$ & \\
\hline \multicolumn{3}{|c|}{ Vascular invasion } & 0.261 & & & 0.045 \\
\hline Absent & 1 & & & 1 & & \\
\hline Present & 1.399 & $(0.779-2.515)$ & & 1.818 & $(1.013-3.262)$ & \\
\hline \multicolumn{3}{|c|}{ Neural invasion } & 0.503 & & & 0.156 \\
\hline Absent & 1 & & & 1 & & \\
\hline Present & 1.199 & $(0.705-2.041)$ & & 1.462 & $(0.865-2.471)$ & \\
\hline \multicolumn{3}{|c|}{ Stage by AJCC7 } & $<0.001$ & & & $<0.001$ \\
\hline I & 1 & & & 1 & & \\
\hline II & 2.260 & $(1.070-4.772)$ & & 2.392 & $(1.147-4.988)$ & \\
\hline III & 5.843 & $(2.830-12.066)$ & & 4.429 & $(2.184-8.983)$ & \\
\hline \multicolumn{3}{|l|}{ PD-L1 } & 0.018 & & & 0.059 \\
\hline Negative & 1 & & & 1 & & \\
\hline Positive & 0.582 & $(0.372-0.910)$ & & 0.651 & $(0.416-1.017)$ & \\
\hline \multicolumn{3}{|l|}{ CD3 } & 0.038 & & & 0.005 \\
\hline Low & 1 & & & 1 & & \\
\hline High & 0.624 & $(0.399-0.976)$ & & 0.538 & $(0.347-0.832)$ & \\
\hline
\end{tabular}

suggests that the density of $\mathrm{CD} 3(+)$ cells, which includes both $\mathrm{CD} 4(+)$ and $\mathrm{CD} 8(+)$ cells, predicts the prognosis of GC patients after curative gastrectomy more precisely than the densities of T-cell subtypes (CD4(+) or CD4(+)). Our observations are consistent with those made in a previous systemic review [35]. In that report on the prognostic potential of TILs in various cancers, $\mathrm{CD} 3(+)$ cells had a lower HR for OS $(0.58 ; 95 \% \mathrm{CI}, 0.43-0.78)$ than CD8(+) cells (HR 0.71; $95 \% \mathrm{CI}, 0.62-0.82)$ or CD4(+) cells (HR $0.82 ; 95 \% \mathrm{CI}, 0.69-0.98)$ did [35]. Considering the results of the previous systemic review and our study, CD3(+) TILs, which include both CD4(+) and CD8(+) cells, may yield more precise information about an immune response against the tumor and about the patient's prognosis. The PD-1(+) cell density in the tumor microenvironment also appeared to be related to survival outcomes, although there was no statistical significance (Fig. 2g, h); this finding is similar to that of a previous study, in which PD-1expressing tumor-infiltrating $\mathrm{T}$ cells were a favorable prognostic biomarker in head and neck cancer [36].

In summary, our study reveals that immunosuppressive proteins are expressed in some patients with GC, and that these patients have distinct clinicopathological characteristics. In tumors, there were strong correlations among the protein expression levels of immunosuppressive markers (PD-L1, CTLA-4, and IDO). However, no correlation was observed between the expression of immunosuppressive proteins in tumors and the density of immune cells in the tumor microenvironment. These results support the idea that immunosuppressive proteins in tumors are induced by some intrinsic tumor-related mechanisms, which need to be further investigated in future studies, rather than being a tumor response to host immunity. PD-L1(+) expression in tumors and a high-CD3 microenvironment were related to favorable survival outcomes, regardless of other clinical factors.

Acknowledgments This study was supported by grant no 02-2013064 from the SNUBH research fund.

\section{References}

1. Hanahan D, Weinberg RA. Hallmarks of cancer: the next generation. Cell. 2011;144:646-74.

2. Klein G, Klein E. Surveillance against tumors-is it mainly immunological? Immunol Lett. 2005;100:29-33.

3. Hoenicke L, Zender L. Immune surveillance of senescent cellsbiological significance in cancer- and non-cancer pathologies. Carcinogenesis. 2012;33:1123-6. 
4. Merelli B, Massi D, Cattaneo L, Mandala M. Targeting the PD1/ PD-L1 axis in melanoma: biological rationale, clinical challenges and opportunities. Crit Rev Oncol Hematol. 2014;89:140-65.

5. Hamid O, Robert C, Daud A, Hodi FS, Hwu WJ, Kefford R, et al. Safety and tumor responses with lambrolizumab (anti-PD-1) in melanoma. N Engl J Med. 2013;369:134-44.

6. Brahmer JR, Tykodi SS, Chow LQ, Hwu WJ, Topalian SL, Hwu P, et al. Safety and activity of anti-PD-L1 antibody in patients with advanced cancer. N Engl J Med. 2012;366:2455-65.

7. Topalian SL, Hodi FS, Brahmer JR, Gettinger SN, Smith DC, McDermott DF, et al. Safety, activity, and immune correlates of anti-PD-1 antibody in cancer. N Engl J Med. 2012;366:2443-54.

8. Robert C, Thomas L, Bondarenko I, O'Day S, JW MD, Garbe C, et al. Ipilimumab plus dacarbazine for previously untreated metastatic melanoma. N Engl J Med. 2011;364:2517-26.

9. Hodi FS, O'Day SJ, McDermott DF, Weber RW, Sosman JA, Haanen JB, et al. Improved survival with ipilimumab in patients with metastatic melanoma. N Engl J Med. 2010;363:711-23.

10. Naito Y, Saito K, Shiiba K, Ohuchi A, Saigenji K, Nagura H, et al. CD8 $+\mathrm{T}$ cells infiltrated within cancer cell nests as a prognostic factor in human colorectal cancer. Cancer Res. 1998;58:3491-4.

11. Schumacher K, Haensch W, Roefzaad C, Schlag PM. Prognostic significance of activated $\mathrm{CD} 8(+) \mathrm{T}$ cell infiltrations within esophageal carcinomas. Cancer Res. 2001;61:3932-6.

12. Nakano O, Sato M, Naito Y, Suzuki K, Orikasa S, Aizawa M, et al. Proliferative activity of intratumoral CD8(+) T-lymphocytes as a prognostic factor in human renal cell carcinoma: clinicopathologic demonstration of antitumor immunity. Cancer Res. 2001;61:5132-6.

13. Anraku M, Cunningham KS, Yun Z, Tsao MS, Zhang L, Keshavjee $\mathrm{S}$, et al. Impact of tumor-infiltrating $\mathrm{T}$ cells on survival in patients with malignant pleural mesothelioma. J Thorac Cardiovasc Surg. 2008;135:823-9.

14. Vergani A, D'Addio F, Jurewicz M, Petrelli A, Watanabe T, Liu $\mathrm{K}$, et al. A novel clinically relevant strategy to abrogate autoimmunity and regulate alloimmunity in NOD mice. Diabetes. 2010;59:2253-64.

15. Sun $\mathrm{J}, \mathrm{Xu} \mathrm{K}, \mathrm{Wu} \mathrm{C}$, Wang $\mathrm{Y}, \mathrm{Hu} \mathrm{Y}, \mathrm{Zhu} \mathrm{Y}$, et al. PD-L1 expression analysis in gastric carcinoma tissue and blocking of tumor-associated PD-L1 signaling by two functional monoclonal antibodies. Tissue Antigens. 2007;69:19-27.

16. Wu C, Zhu Y, Jiang J, Zhao J, Zhang XG, Xu N. Immunohistochemical localization of programmed death-1 ligand-1 (PD-L1) in gastric carcinoma and its clinical significance. Acta Histochem. 2006;108:19-24.

17. Soliman H, Khalil F, Antonia S. PD-L1 expression is increased in a subset of basal type breast cancer cells. PLoS One. 2014;9:e88557.

18. Taube JM, Anders RA, Young GD, Xu H, Sharma R, McMiller $\mathrm{TL}$, et al. Colocalization of inflammatory response with B7-h1 expression in human melanocytic lesions supports an adaptive resistance mechanism of immune escape. Sci Transl Med. 2012;4:127ra37.

19. Gadiot J, Hooijkaas AI, Kaiser AD, van Tinteren H, van Boven $\mathrm{H}$, Blank C. Overall survival and PD-L1 expression in metastasized malignant melanoma. Cancer. 2011;117:2192-201.

20. Brunet JF, Denizot F, Luciani MF, Roux-Dosseto M, Suzan M, Mattei MG, et al. A new member of the immunoglobulin superfamily-CTLA-4. Nature. 1987;328:267-70.

21. Gao YF, Peng RQ, Li J, Ding Y, Zhang X, Wu XJ, et al. The paradoxical patterns of expression of indoleamine 2,3-dioxygenase in colon cancer. J Transl Med. 2009;7:71.
22. Pan K, Wang H, Chen MS, Zhang HK, Weng DS, Zhou J, et al. Expression and prognosis role of indoleamine 2,3-dioxygenase in hepatocellular carcinoma. J Cancer Res Clin Oncol. 2008;134:1247-53.

23. Hamanishi J, Mandai M, Iwasaki M, Okazaki T, Tanaka Y, Yamaguchi K, et al. Programmed cell death 1 ligand 1 and tumorinfiltrating $\mathrm{CD} 8+\mathrm{T}$ lymphocytes are prognostic factors of human ovarian cancer. Proc Natl Acad Sci USA. 2007;104:3360-5.

24. Contardi E, Palmisano GL, Tazzari PL, Martelli AM, Fala F, Fabbi M, et al. CTLA-4 is constitutively expressed on tumor cells and can trigger apoptosis upon ligand interaction. Int $\mathrm{J}$ Cancer. 2005;117:538-50.

25. Astigiano S, Morandi B, Costa R, Mastracci L, D'Agostino A, Ratto GB, et al. Eosinophil granulocytes account for indoleamine 2,3-dioxygenase-mediated immune escape in human non-small cell lung cancer. Neoplasia. 2005;7:390-6.

26. Pistillo MP, Tazzari PL, Palmisano GL, Pierri I, Bolognesi A, Ferlito F, et al. CTLA-4 is not restricted to the lymphoid cell lineage and can function as a target molecule for apoptosis induction of leukemic cells. Blood. 2003;101:202-9.

27. Uyttenhove C, Pilotte L, Theate I, Stroobant V, Colau D, Parmentier $\mathrm{N}$, et al. Evidence for a tumoral immune resistance mechanism based on tryptophan degradation by indoleamine 2,3dioxygenase. Nat Med. 2003;9:1269-74.

28. Thompson RH, Kuntz SM, Leibovich BC, Dong H, Lohse CM, Webster WS, et al. Tumor B7-H1 is associated with poor prognosis in renal cell carcinoma patients with long-term follow-up. Cancer Res. 2006;66:3381-5.

29. Thompson RH, Gillett MD, Cheville JC, Lohse CM, Dong H, Webster WS, et al. Costimulatory B7-H1 in renal cell carcinoma patients: indicator of tumor aggressiveness and potential therapeutic target. Proc Natl Acad Sci USA. 2004;101:17174-9.

30. Zang X, Thompson RH, Al-Ahmadie HA, Serio AM, Reuter VE, Eastham JA, et al. B7-H3 and B7× are highly expressed in human prostate cancer and associated with disease spread and poor outcome. Proc Natl Acad Sci USA. 2007;104:19458-63.

31. Karim R, Jordanova ES, Piersma SJ, Kenter GG, Chen L, Boer $\mathrm{JM}$, et al. Tumor-expressed B7-H1 and B7-DC in relation to PD$1+$ T-cell infiltration and survival of patients with cervical carcinoma. Clin Cancer Res. 2009;15:6341-7.

32. Parsa AT, Waldron JS, Panner A, Crane CA, Parney IF, Barry JJ, et al. Loss of tumor suppressor PTEN function increases B7-H1 expression and immunoresistance in glioma. Nat Med. 2007;13:84-8.

33. Zhang L, Conejo-Garcia JR, Katsaros D, Gimotty PA, Massobrio $\mathrm{M}$, Regnani G, et al. Intratumoral T cells, recurrence, and survival in epithelial ovarian cancer. N Engl J Med. 2003;348:203-13.

34. Galon J, Costes A, Sanchez-Cabo F, Kirilovsky A, Mlecnik B, Lagorce-Pages $\mathrm{C}$, et al. Type, density, and location of immune cells within human colorectal tumors predict clinical outcome. Science. 2006;313:1960-4.

35. Gooden MJ, de Bock GH, Leffers N, Daemen T, Nijman HW. The prognostic influence of tumour-infiltrating lymphocytes in cancer: a systematic review with meta-analysis. $\mathrm{Br} \mathrm{J}$ Cancer. 2011;105:93-103.

36. Badoual C, Hans S, Merillon N, Van Ryswick C, Ravel P, Benhamouda N, et al. PD-1-expressing tumor-infiltrating $\mathrm{T}$ cells are a favorable prognostic biomarker in HPV-associated head and neck cancer. Cancer Res. 2013;73:128-38. 